\title{
Alternancia Escolar: un reto para garantizar la salud y la vida de la comunidad educativa
}

Como citar este artículo:

Oróstegui Arenas Myriam, Bautista Lorenzo Leonelo Enrique, Martínez Vega Ruth Aralí, Sosa Ávila Luis Miguel, Vera Cala Lina María, Rodríguez Villamizar Laura Andrea, Herrera Galindo Víctor Mauricio. Alternancia Escolar: un reto para garantizar la salud y la vida de la comunidad educativa. Revista Cuidarte. 2021;12(2):e.2244

http://dx.doi.org/10.15649/cuidarte.2244

Revista Cuidarte Rev Cuid. May - Ago 2021; 12(2): e2244 doij http://dx.doi.org/10.15649/cuidarte.2244

E-ISSN: 2346-3414

(1) Myriam Oróstegui Arenas ${ }^{1}$

(1) Leonelo Enrique Bautista Lorenzo $^{2}$

(1) Ruth Aralí Martínez Vega ${ }^{3}$

(1) Luis Miguel Sosa Ávila ${ }^{4}$

(1) Lina María Vera Cala

(1) Laura Andrea Rodríguez Villamizar 6

(1) Víctor Mauricio Herrera Galindo ${ }^{7}$

1 Enfermera, Magister en Epidemiologia, Profesora Emérita Universidad Industrial de Santander, Santander Colombia.

E-mail:_ciepi_uis@hotmail.com Autora de Correspondencia

2 Médico, PhD en Epidemiologia, Profesor Universidad de Madison, Estados Unidos. Email: lebautista@ wisc.edu

3 Médica, PhD en Epidemiologia. Profesora Universidad de Santander, Santander, Colombia. E-mail: rutharam@yahoo.com

4 Médico, Esp en Pediatria, Infectólogo Profesor Universidad Industrial de Santander, Santander, Colombia. E-mail: Imsosavi@uis.edu.co

5 Médica, PhD en Epidemiologia. Profesora Universidad Industrial de Santander, Santander, Colombia. E-mail: limavera@uis.edu.co

6 Médica, PhD en Epidemiologia. Profesora Universidad Industrial de Santander, Santander, Colombia. E-mail: laurovi@uis.edu.co

7 Médico, PhD en Epidemiologia. Profesor Universidad Autónoma de Bucaramanga, Santander, Colombia. E-mail:vherrera@unab.edu.co
La inesperada llegada de la pandemia, enfrentó a todo el mundo a cambios drásticos en la forma de vida que se disfrutaba en épocas de normalidad, obligando a la población a diferentes tipos de confinamientos. Estos trajeron consigo el cierre de casi todos los sectores y en consecuencia la interrupción de muchos servicios esenciales, como las intervenciones en el sector educativo. Muchas de estas intervenciones estaban dirigidas, además de a la educación misma, a brindar protección a los escolares, detectar condiciones de riesgo de violencia intrafamiliar, maltrato y trabajo infantil, suministrar raciones alimenticias, vigilar el cumplimiento del esquema de vacunación y a evitar la deserción escolar, entre otras.

Aunque los escolares no son el grupo más afectado por COVID-19, un reciente estudio de UNICEF realizado en 87 países revela que, en noviembre de 2020, los niños y los adolescentes representaron el $11 \%$ del total de infecciones ${ }^{1}$, mientras que, en Colombia, la proporción de casos pediátricos (menores de 18 años) se estima entre $7-8 \%$ y representan el $0.025 \%$ del total de fallecimientos ${ }^{2}$.

Aunque se ha reportado que el riesgo de infección en escolares es menorque el del personal docenteyadministrativo en instituciones de educación 3, esta discrepancia pudiera estar sobreestimada debido a una menor probabilidad de detección considerando el curso predominantemente asintomático de la infección en niños. Sin embargo, COVID-19 puede ser una enfermedad severa en los menores, contribuyendo no solo al número de ingresos a unidades de cuidados intensivo sino al de casos fatales ${ }^{3}$.

Recibido: 4 de mayo de 2021

Aceptado: 6 de mayo de 2021

Publicado: 31 de mayo de 2021 $\square *$ Correspondencia Myriam Oróstegui Arenas ${ }^{1}$ E-mail:ciepi_uis@hotmail.com 
En términos de la transmisión, un estudio conducido en población infantil evidenció que esta ocurre con mayorfrecuenciaen elentornofamiliar, relacionándose directamente con el estadio de enfermedad del caso índice: comparados con casos asintomáticos aquellos en etapa pre sintomática fueron responsables del doble de casos secundarios $(1 / 3 \text { versus } 1 / 6)^{4}$. Esto indica que los niños infectados constituyen una fuente con evidencia de mayor transmisión en el nivel de secundaria comparado con el de primaria ${ }^{5}$, así como para los miembros del personal docente y administrativo de sus escuelas. Estos últimos, a su vez generarían nuevos casos de infección en la comunidad estudiantil y dada cuenta de su mayor movilidad, también en la población general.

La importancia que la socialización tiene en los procesos de enseñanza-aprendizaje y en el desarrollo psicológico de los niños, niñas, adolescentes y jóvenes es ampliamente reconocida. Además, es conocido el impacto beneficioso que tienen las actividades educativas en la salud física y mental y en general, en la probabilidad que nuestros niños alcancen todo su potencial y tengan una vida plena. En esta dirección, y ajustándose a la coyuntura, el sector educativo ha tenido que instaurar nuevas estrategias, pasando a la modalidad virtual (asistida por las tecnologías de la información y las comunicaciones - TICs) y a diferentes modelos de alternancia escolar.

La alternancia se ofrece en diferentes modalidades como la híbrida, en la que es posible que un grupo de estudiantes reciban de manera presencial la clase y el resto lo hagan de manera virtual en forma sincrónica, con opciones de participación en ambos espacios. Otra estrategia es el desarrollo de la clase presencial y de manera asincrónica el desarrollo de actividades virtuales en casa, es decir, con grupos de estudiantes que tendrán asistencia a la institución y trabajo virtual o so-lamente asistencia a la institución. Las instituciones que no acojan la alternancia continuarán con su oferta pedagógica en la modalidad virtual. Cualquiera de los modelos de alternancia que las instituciones adopten trae consigo la presencialidad y, por lo tanto, la necesidad de realizar acciones para la disminución del riesgo, la identificación de casos (sintomáticos y asintomáticos) y sus contactos y el seguimiento y aislamiento, cuando ello se requiera.

Nadie niega la importancia del regreso a la escuela, pero ésta debe hacerse en condiciones seguras que garanticen la protección de la salud y la vida de escolares, maestros, personal administrativo y de apoyo y de sus familias. Para lograr este objetivo se requiere adherencia y adaptación en cada institución educativa de los protocolos de bioseguridad emanados de los ministerios deeducación y salud. Además, se requiere tener en cuenta la situación epidemiológica actual del municipio o área metropolitana y de la evolución de la epidemia en la población local y la comunidad educativa institucional. Esto último, el conocimiento cabal de la situación epidemiológica local e institucional es crucial para poder decidir responsablemente cuando se abre o se cierra una institución educativa, a fin de preservar la salud, el bienestar y la vida de sus integrantes.
El conocimiento cabal de la situación epidemiológica local e institucional es crucial para poder decidir responsablemente cuando se abre o se cierra una institución educativa, a fin de preservar la salud, el bienestar y la vida de sus integrantes. 
En particular, al momento de tomar estas decisiones, es esencial tener en cuenta los siguientes aspectos:

- La situación de la transmisión y la velocidad de contagio del SARS-CoV-2 en la localidad y cómo esto afectará el entorno educativo

- El nivel de capacidad de los servicios de salud y especialmente las EPS para detectar y aislar casos sintomáticos y asintomáticos; detectar brotes o conglomerados de casos; identificar y seguir contactos de los casos; y establecer cercos epidemiológicos para detener la transmisión de la enfermedad dentro de la institución educativa.

- En qué medida la infección y enfermedad en familiares de los estudiantes, profesores y otro personal de la institución, constituye un factor de riesgo para los niños y viceversa.

- Dada la dinámica de la transmisión, los protocolos de bioseguridad por sí mismos, no garantizan la reducción del riesgo, si no se acompañan del cumplimiento estricto de los mismos, del acondicionamiento de las instituciones educativas para garantizar ambientes seguros, y de un análisis técnico, permanente y veraz de las condiciones de la pandemia en la localidad.

- Garantizar la ventilación adecuada y los recambios de aire, de los diferentes espacios de las instituciones educativas especialmente en las áreas cerradas, es un factor de especial importancia para disminuir la transmisión del virus. Por lo tanto, este aspecto es un asunto clave en la adecuación de las instituciones educativas.

- La decisión de abrir una institución de educación, debe estar precedida de un trabajo conjunto entre los escolares, los padres, los maestros, el personal directivo, y el personal de salud, con el fin de fijar los lineamientos básicos de las decisiones y acciones tanto en los asuntos de salud, como de protección de los escolares y del personal y sus familias, la coordinación con las entidades de salud y la anticipación de la mayoría de las situaciones que puedan derivarse de los procesos de apertura.

- La institución educativa debe definir, con el apoyo del sector salud, los indicadores que les permitan decidir en qué momento la institución debe cerrarse, bien sea de manera parcial o total, dada la existencia de casos en una burbuja (grupos estables de convivencia) o en varias burbujas.

- Además de los aspectos relacionados con minimizar el riesgo y disminuir la transmisión, en el proceso de apertura deben contemplarse otros aspectos tales como: garantizar el acceso a la educación a distancia a todos los escolares de las poblaciones distantes o rurales, marginadas, de escasos recursos, con discapacidad y cuyos familiares pertenecen a grupos de alto riesgo.

- Garantizar mecanismos pedagógicos que les permitan a los escolares, superar la brecha en que pudieron haber caído, por las dificultades de acceso a la educación virtual.

- Proveer la asistencia en salud y nutrición a los escolares, así como la atención a las niñas y adolescentes para la prevención del embarazo, el acoso y el abuso sexual

Ante la situación tan cambiante de la pandemia, es necesario tomar las decisiones de la apertura de las instituciones, en medio de gran incertidumbre. Son muchos los retos que el sector educativo tiene que superar. Pero en la planeación y toma de decisiones, como bien lo enuncia la UNICEF, "la respuesta debe servir de catalizador para mejorar los resultados del aprendizaje, hacer más equitativo el acceso a la enseñanza y fortalecer la protección, la salud y la seguridad de los niños" ${ }^{\prime}$. En consecuencia, si bien es prioritario el regreso a la presencialidad especialmente en los niveles de educación básica, deberán primar el principio de la protección y bienestar 
de los niños y los principios éticos, por encima de cualquier interés político o económico, del ejercicio de autoridad, o de una falsa sensación de seguridad.

Conflicto de intereses: Los autores declaran no tener conflicto de intereses.

\section{Referencias}

1. Unicef. Evitar una generación perdida a causa de la COVID-19: Un plan de seis puntos para responder, recuperarse y reimaginar un mundo para todos los niños después de la pandemia. Consultado en: https://www.unicef.org/media/87156/file/Evitar-una-generacion-perdidacausa-covid-2020.pdf

2. INS. https://www.ins.gov.co/Noticias/paginas/coronavirus.aspx

3. Liu C, He Y, Liu L, Li F, Shi Y. Children with COVID-19 behaving milder may challenge the public policies: a systematic review and meta-analysis. BMC Pediatrics. 2020:410. https://doi.org/10.1186/s12887-020-02316-1

4. Thompson H, Mousa A, Dighe A, Fu H, Arnedo-Pena A. Barrett P. et al. Severe Acute Respiratory Syndrome Coronavirus 2 (SARS-CoV-2) Setting-specific Transmission Rates: A Systematic Review and Meta-analysis. Clinical Infectious Diseases. 2021: ciab100. https://doi.org/10.1093/cid/ciab100

5. Goldstein E, Lipsitch M, Cevik M. On the Effect of Age on the Transmission of SARS-CoV-2 in Households, Schools, and the Community. J Infect Dis. 2021;223(3):362-369. https://doi.org/10.1093/infdis/jiaa691

6. Ismail SA, Saliba V, Lopez Bernal J, Ramsay ME, Ladhani SN. SARS-CoV-2 infection and transmission in educational settings: a prospective, cross-sectional analysis of infection clusters and outbreaks in England. Lancet Infect Dis. 2021;21(3):344-353.

https://doi.org/10.1016/S1473-3099(20)30882-3 